\title{
RANDOM BIPARTITE POSETS AND EXTREMAL PROBLEMS
}

\author{
CSABA BIRÓ, PETER HAMBURGER, H. A. KIERSTEAD, ATTILA PÓR, \\ WILLIAM T. TROTTER, AND RUIDONG WANG
}

\begin{abstract}
Previously, Erdős, Kierstead and Trotter [5] investigated the dimension of random height 2 partially ordered sets. Their research was motivated primarily by two goals: (1) analyzing the relative tightness of the Füredi-Kahn upper bounds on dimension in terms of maximum degree; and (2) developing machinery for estimating the expected dimension of a random labeled poset on $n$ points. For these reasons, most of their effort was focused on the case $0<p \leq 1 / 2$. While bounds were given for the range $1 / 2 \leq p<1$, the relative accuracy of the results in the original paper deteriorated as $p$ approaches 1 .

Motivated by two extremal problems involving conditions that force a poset to contain a large standard example, we were compelled to revisit this subject, but now with primary emphasis on the range $1 / 2 \leq p<1$. Our sharpened analysis shows that as $p$ approaches 1 , the expected value of dimension increases and then decreases, answering in the negative a question posed in the original paper. Along the way, we apply inequalities of Talagrand and Janson, establish connections with latin rectangles and the Euler product function, and make progress on both extremal problems.
\end{abstract}

\section{INTRODUCTION}

This paper is concerned primarily with the combinatorics of finite partially ordered sets, also called posets, but to motivate our line of research, we start with a brief discussion of analogous questions for graphs. For a graph $G$, let $\omega(G)$ denote the clique number of $G$, the maximum number of vertices in a complete subgraph of $G$. Also, let $\chi(G)$ denote the chromatic number of $G$, the least number of colors required for a proper coloring of $G$. Let $N$ denote the set of positive integers, and when $n \in N$, we write $[n]$ for $\{1, \ldots, n\}$.

Let $n \in N$, and let $G$ be a graph on $n$ vertices. Then we have the trivial inequality $\omega(G) \leq \chi(G) \leq n$. Furthermore, if $\chi(G)=n$, then $\omega(G)=n$. We then ask whether these statements are "stable," i.e., if $G$ is a graph on $n$ vertices and $\chi(G)$ is close to $n$, must $\omega(G)$ also be close to $n$ ? More formally:

Question 1.1 (Question 1 for Graphs). Does there exist a function $f: N \rightarrow N$ such that for every $c \in N$, if $n>f(c), G$ is a graph on $n$ vertices, and $\chi(G) \geq n-c$, then $\omega(G) \geq n-f(c)$ ?

The answer to Question 1 for graphs is easily seen to be yes, since we may take $f(c)=2 c$. To see that this function satisfies the desired property, we simply carry out the following iterative process: While $G$ is not a complete graph, choose two

Date: February 21, 2020.

2010 Mathematics Subject Classification. 06A07, 05C35.

Key words and phrases. Poset, bipartite poset, dimension, standard example. 
non-adjacent vertices and remove them. Each such operation decreases the size of the graph by 2 but lowers the chromatic number by at most 1 . So the operation must halt in at most $c$ steps.

Here is a second question of a related nature.

Question 1.2 (Question 2 for Graphs). For integers $k$ and $n$ with $k \geq 2$ fixed and $n \rightarrow \infty$, what is the maximum value $g(k, n)$ of $\chi(G)$ among all graphs $G$ on $n$ vertices with $\omega(G)<k$ ?

For example, when $k=3$, a graph $G$ with $\omega(G)<3$ is said to be triangle-free, and it is well known that $g(3, n)$, the maximum chromatic number of a triangle-free graph on $n$ vertices, is $\Theta(\sqrt{n / \log n})$.

The primary goal of this paper is to investigate analogous questions for posets. We assume that readers are familiar with basic notation and terminology for posets, including comparable and incomparable pairs of points; chains and antichains; minimal and maximal elements; and linear extensions. Beyond these basics, we will include all essential notation and terminology for the results presented in this paper.

To develop the poset analogue of chromatic number, we have the following definitions. Let $P$ be a poset. A non-empty family $\mathcal{F}=\left\{L_{1}, \ldots, L_{d}\right\}$ of linear extensions of $P$ is called a realizer of $P$ when $x \leq y$ in $P$ if and only if $x \leq y$ in $L_{j}$ for each $j \in[d]$. Dushnik and Miller [4] defined the dimension of a poset $P$, denoted $\operatorname{dim}(P)$, as the least positive integer $d$ for which there is a realizer $\mathcal{F}=\left\{L_{1}, \ldots, L_{d}\right\}$ of $P$. Analogies between dimension for posets and chromatic number for graphs have been widely studied, and indeed the book chapter [14] is devoted entirely to this topic.

For the poset analogue of a clique, we have the following construction. For an integer $d \geq 2$, let $S_{d}$ be the height 2 poset with $\operatorname{Min}\left(S_{d}\right)=\left\{a_{1}, \ldots, a_{d}\right\}, \operatorname{Max}\left(S_{d}\right)=$ $\left\{a_{1}^{\prime}, \ldots, a_{d}^{\prime}\right\}$ and $a_{i}<a_{j}^{\prime}$ in $S_{d}$ if and only if $i \neq j$. Clearly, $\operatorname{dim}\left(S_{d}\right)=d$, and posets in the family $\left\{S_{d}: d \geq 2\right\}$ are called standard examples. For a poset $P$, we define the standard example number of $P$, denoted $\operatorname{se}(P)$, as follows. Set $\operatorname{se}(P)=1$ if $P$ does not contain a subposet isomorphic to the standard example $S_{2}$; otherwise $\operatorname{se}(P)$ is the largest $d \geq 2$ for which $P$ contains a subposet isomorphic to the standard example $S_{d}$. We then have the trivial inequality $\operatorname{dim}(P) \geq \operatorname{se}(P)$. As is well known, for every $d \geq 2$, there is a poset $P$ with $\operatorname{se}(P)=1$ and $\operatorname{dim}(P)=d$. Nevertheless, it is of interest to study classes of posets where large dimension requires large standard example number.

When $n \in N$ and $G$ is a graph on $n$ vertices, the inequality $\chi(G) \leq n$ is trivial, as is the assertion that the inequality is tight only when $G$ is a complete graph on $n$ vertices. The analogous results for posets are more substantive. Hiraguchi 9 proved that if $n \geq 2$ and $P$ is a poset on $2 n+1$ points, then $\operatorname{dim}(P) \leq n$. Kimble 11$]$ proved that if $n \geq 4$, this inequality is tight only when $P$ contains the standard example $S_{n}$. For the poset analogue of Question 1 for graphs, we then have:

Question 1.3 (Question 1 for Posets). Does there exist a function sa $: N \rightarrow N$ such that for every $c \in N$, if $n>\mathrm{sa}(c), P$ is a poset on $2 n+1$ points, and $\operatorname{dim}(P) \geq n-c$, then $\operatorname{se}(P) \geq n-\operatorname{sa}(c)$ ?

In stating Question 1 for posets, we use the notation sa(c) to remind readers that we are discussing "stability analysis." Unlike the situation with graphs, we

\footnotetext{
${ }^{1}$ We refer the reader to the discussion in [14] about subtleties of this proof, and we note that it does not hold when $n=2$ or when $n=3$.
} 
know of no elementary argument to show that the function sa $(c)$ is well defined, ignoring any issue of how fast $\mathrm{sa}(c)$ would have to grow in terms of $c$. However, in [3], Biró, Hamburger, Pór and Trotter showed the function sa $(c)$ is well defined and satisfies $\mathrm{sa}(c)=O\left(c^{2}\right)$. Also, they gave a construction using finite projective planes to show that $\mathrm{sa}(c)=\Omega\left(c^{4 / 3}\right)$. The research for this paper began with the challenge of finding the correct exponent on $c$ in the function sa(c), knowing that the answer is in the interval $[4 / 3,2]$. In this paper, we will raise the lower bound on this interval to $3 / 2$.

For the poset analogue of Question 2 for graphs, we have:

Question 1.4 (Question 2 for Posets). For integers $d$ and $n$ with $d \geq 2$ fixed and $n \rightarrow \infty$, what is the maximum value $f(d, n)$ of $\operatorname{dim}(P)$ among all posets $P$ on $n$ points with $\operatorname{se}(P)<d$ ?

Question 2 for posets was first posed in [13], and then referenced again in [5]. Here we obtain a better result, and we remove the requirement that $d$ be large. For historical reasons, the value of $f(2, n)$ has been studied-albeit with different notation and terminology - for many years. No doubt this results from the fact that the class of posets with standard example number 1 is the class of interval orders. Combining results of several authors (see the discussion in [3]), the value of $f(2, n)$ can be determined to within an additive error of at most 5 . However, as a crude estimate, we have

$$
f(2, n)=\lg \lg n+(1 / 2+o(1)) \lg \lg \lg n .
$$

For a fixed value of $d \geq 3$, Biró, Hamburger and Pór [2] proved that $f(d, n)=$ $o(n)$, but this leaves open the possibility that $f(d, n)$ behaves in the same slowgrowing manner as $f(2, n)$. However, we will show in Section 6 that there is a positive constant $\alpha_{d}$ so that $f(d, n)=\Omega\left(n^{\alpha_{d}}\right)$.

1.1. Links with Random Bipartite Posets. Working on Question 1 for posets led us to revisit the following model for a random bipartite poset, introduced and studied by Erdős, Kierstead and Trotter [5]. Let $n \in N$ and fix disjoint sets $A$ and $A^{\prime}$, each of size $n$. Then $\Omega(n, p)$ denotes the probability space consisting of posets $P$ such that (1) the ground set of $P$ is $A \cup A^{\prime}$ with $A \subseteq \operatorname{Min}(P)$ and $A^{\prime} \subseteq \operatorname{Max}(P)$; and (2) for a pair $\left(a, a^{\prime}\right) \in A \times A^{\prime}$, set $\mathbb{P}\left(a<a^{\prime}\right.$ in $\left.P\right)=p$, (in general, $p$ is a function of $n$ ) with events corresponding to distinct pairs independent.

To place this work in historical perspective, we give here a brief overview of key results, beginning with a discussion of upper bounds on dimension. For a poset $P$ (of arbitrary height), let $\Delta_{U}(P)$ denote the maximum size of sets of the form $U_{P}(x)=\{y \in P: x \leq y$ in $P\}$ taken over all elements $x \in P$. Analogously, $\Delta_{D}(P)$ is the maximum size of sets of the form $D_{P}(x)=\{z \in P: z \leq x$ in $P\}$. Then set $\Delta(P)=\max \left\{\Delta_{U}(P), \Delta_{D}(P)\right\}$. In [8], Füredi and Kahn proved that if $\Delta(P)=k$, then $\operatorname{dim}(P)<50 k \log ^{2} k$.

The first inequality in the following theorem is due to Füredi and Kahn [8]. The second is a quite recent result of Scott and Wood [12] improving the bound from [8] cited immediately above. Readers may note that the Lovász local lemma [6] was used in both [8] and [12]. 
Theorem 1.5. If $u=\Delta_{U}(P), k=\Delta(P)$ and $|P|=n$, then

$$
\begin{aligned}
& \operatorname{dim}(P)<1+2(u+1) \log n \quad \text { and } \\
& \operatorname{dim}(P)<k \log ^{1+o(1)} k
\end{aligned}
$$

Continuing with upper bounds, as is well known, almost all labeled posets on $n$ points have the following structure: $P$ is the union $\operatorname{Min}(P) \cup A \cup \operatorname{Max}(P)$ of three disjoint antichains; the size of $A$ is $(1 / 2 \pm o(1)) n$; both $\operatorname{Min}(P)$ and $\operatorname{Max}(P)$ have size $(1 / 4 \pm o(1)) n$; and $x<y$ in $P$ whenever $x \in \operatorname{Min}(P)$ and $y \in \operatorname{Max}(P)$. It is then straightforward to show that there is a constant $c_{1}>0$ such that almost all labeled posets on $n$ points have dimension at most $n / 4-c_{1} n / \log n$.

Turning to lower bounds, it is more challenging to find good lower bounds in either of these two settings. For example, no explicit construction is known for a poset $P$ with $\Delta(P)=k$ and $\operatorname{dim}(P)>k+1$ for any value of $k$. Also, simple counting only shows that almost all labeled posets on $n$ elements have dimension $\Omega(n / \log n)$.

The following lower bounds are proved in [5. In stating these bounds, we use the standard abbreviation a.a.s. for asymptotically almost surely.

Theorem 1.6. For every $\epsilon>0$, there exists positive constants $\delta_{1}, \delta_{2}, \delta_{3}$ so that a.a.s.,

$$
\operatorname{dim}(P)> \begin{cases}\delta_{1} p n \log p n & \text { if } n^{-1+\epsilon}<p \leq 1 / \log n, \text { and } \\ \max \left\{\delta_{2} n, n-\delta_{3} n /(p \log n)\right\} & \text { if } 1 / \log n \leq p<1-n^{-1+\epsilon}\end{cases}
$$

The first inequality in Theorem [1.6 shows that the two upper bounds in Theorem 1.5 are essentially best possible - although there remains an $o(\log k)$ multiplicative gap for the second. The second inequality in Theorem 1.6 was used in [5] to show that is a positive constant $c_{2}>0$ so that almost all labeled posets on $n$ elements have dimension greater than $n / 4-c_{2} n / \log n$.

When $n^{-1} \log ^{2} n<p \leq 1 / \log n$, good upper bounds on the expected value of the dimension of a poset $P \in \Omega(n, p)$ are provided by Theorem 1.5. since $\Delta(P)$ and $\Delta_{U}(P)$ are sharply concentrated around $p n$. For the range $1 / \log n \leq p<1$, the following upper bound is given in 5 .

Theorem 1.7. If $\epsilon>0$ and $1 / \log n \leq p<1$, then a.a.s.,

$$
\operatorname{dim}(P) \leq n-\frac{n \log (1 / p)}{(2+\epsilon) \log n}
$$

The improvements we make here will all be for the range $p \geq 1 / 2$. Accordingly, we extract the following upper and lower bounds from Theorems 1.7 and 1.6 for this range. Note that the modest improvement in the upper bound results from the narrowing of the range on $p$. Note also that $p$ is bounded away from 1 for the lower bound.

Corollary 1.8 (Old Upper and Lower Bounds). Suppose $1 / 2 \leq p<1$, then a.a.s.,

$$
\operatorname{dim}(P)<n-\frac{n \log (1 / p)}{2 \log n} .
$$

Furthermore, for every $\epsilon>0$, there exists $\delta>0$ so that $p<1-n^{-1+\epsilon}$, then a.a.s.,

$$
\operatorname{dim}(P)>n-\frac{\delta n}{\log n} .
$$


In discussing the merits of our new results, the old upper and lower bounds given in Corollary 1.8 will be the basis of comparison.

To make the connection with Question 1 for posets concrete, let $p=1-n^{-1 / 2}$. If $P \in \Omega(n, p)$, then a.a.s., $\operatorname{se}(P)=O(\sqrt{n} \log n)$. Furthermore, the old upper bound in Corollary 1.8 implies that a.a.s., $\operatorname{dim}(P) \leq n-\sqrt{n} /(2 \log n)$. It is easy to see that if this upper bound is tight, up to a poly-log multiplicative factor on the difference $n-\operatorname{dim}(P)$, then the exponent 2 on $c$ in the function $\mathrm{sa}(c)$ is correct.

However, the old lower bound in Corollary 1.8 only asserts that there is a constant $\delta$ such that a.a.s., $\operatorname{dim}(P) \geq n-\delta n / \log n$. This inequality is enough to prove that $\mathrm{sa}(c)=\Omega(c \log c)$, but we already had a constructive proof of an even better lower bound. This shortcoming was the launching point for revisiting the subject of dimension for random bipartite posets, but now with the specific goal of obtaining better bounds when $p \geq 1 / 2$.

The bridges to Question 2 for posets were not clear at the outset of our research but came into view as better bounds and connections to Question 1 unfolded. These details will become clear later in the paper.

1.2. Statement of Improved Bounds for Random Bipartite Posets. To avoid sporadic effects when $p$ is very close to 1 , we assume $p \leq 1-n^{-1} \log ^{2} n$. Consistent with modern research in combinatorics, we will typically treat a quantity like $(1-p) n / \log n$ as if it is an integer when it tends to infinity with $n$. The minor errors this approach introduces can be easily repaired.

For the range $1 / 2 \leq p \leq 1-n^{-1} \log ^{2} n$, many of the results and arguments are more naturally phrased in terms of the complementary parameter $q=1-p$. Accordingly, for the balance of the paper, the symbol $p$ will be used exclusively as a quantity (usually a function of $n$ ) from the interval $[0,1]$, while $q$ will always be $1-p$. Some of the proofs of our new results are extensions and refinements of arguments appearing in [5] and [3], but most of our results require entirely new approaches. In particular, we will apply some second moment methods, Talagrand's inequality and Janson's inequality. None of these tools were used in [5] or in [3].

Our improved upper bounds, stated below in comprehensive form, involve the well-studied Euler product function $\phi(q)=\prod_{i=1}^{\infty}\left(1-q^{i}\right)$.

Theorem 1.9. Suppose $n^{-1} \log ^{2} n \leq q \leq 1 / 2,0<\epsilon<1$ and $z=n^{2} q \log (1 / \phi(q))$. Then a.a.s.,

$$
\operatorname{dim}(P)< \begin{cases}n-(2-\epsilon) \log (q n) / q & \text { if } n^{-1} \log ^{2} n \leq q \leq n^{-1 / 2} \log n . \\ n-q n /(2 \log (q n)) & \text { if } n^{-1 / 2} \log n<q \leq n^{-1 / 3} \\ n-n \log (1 / \phi(q)) / \log z & \text { if } n^{-1 / 3}<q \leq 1 / 2 .\end{cases}
$$

In the discussion to follow, we will refer to these three inequalities as New Upper Bounds (1), (2), and (3). New Upper Bounds (2) and (3) are minor improvements relative to the old upper bound. For example, when $q=1 / 2$, the old upper bound asserts that a.a.s., $\operatorname{dim}(P)<n-0.346 n / \log n$. On the other hand, New Upper Bound (3) improves this to a.a.s., $\operatorname{dim}(P)<n-0.621 n / \log n$. When $q=n^{-1 / 2} \log n$, the old upper bound asserts that a.a.s., $\operatorname{dim}(P)<n-\sqrt{n} / 2$, while New Upper Bound (2) improves this to a.a.s., $\operatorname{dim}(P)<n-\sqrt{n}$.

New Upper Bound (1) is substantially better than the old bound. For example, when $q=n^{-\alpha}$ and $0<\alpha<1 / 2$, the old upper bound asserts that a.a.s., $\operatorname{dim}(P)<$ 
$n-n^{1-\alpha} / 2 \log n$, while New Upper Bound (1) improves this to a.a.s., $\operatorname{dim}(P)<$ $n-(1-\alpha) n^{\alpha} \log n$.

Here is a statement, again in comprehensive form, of our improved lower bounds.

Theorem 1.10. Suppose $n^{-1} \log ^{2} n \leq q \leq 1 / 2$ and $0<\epsilon<1$. If $q \geq n^{-1 / 4} \log ^{3} n$, set $z=\log n+4 \log q-8 \log \log n$. Then a.a.s.,

$$
\operatorname{dim}(P)> \begin{cases}n-(2+\epsilon) \log (q n) / q & \text { if } n^{-1} \log ^{2} n \leq q \leq n^{-4 / 5} . \\ n-32(n \log n / q)^{1 / 2} & \text { if } n^{-4 / 5} \leq q \leq(32)^{1 / 3} n^{-1 / 3} \log ^{1 / 3} n . \\ n-8 q n & \text { if }(32)^{1 / 3} n^{-1 / 3} \log ^{1 / 3} n \leq q \leq \frac{1}{8} n^{-1 / 4} \log ^{3} n . \\ n-24 q n / z & \text { if } \frac{1}{8} n^{-1 / 4} \log ^{3} n \leq q \leq 1 / 2 .\end{cases}
$$

In the discussion to follow, we will refer to these inequalities as New Lower Bounds (1) through (4). In the range $n^{-1} \log ^{2} n \leq q \leq 1 / 2$, our bounds show that a.a.s., $\operatorname{dim}(P) \geq(1-o(1)) n$. So the accuracy of our upper and lower bounds should be judged on the quantity $n-\operatorname{dim}(P)$. For this quantity, our upper and lower bounds differ by a multiplicative factor that is $1+o(1)$ when $n^{-1} \log ^{2} n \leq q \leq n^{-4 / 5}$, and by $O(\log n)$ when $n^{-1 / 3} \leq q \leq 1 / 2$. In [5], it was asked whether the expected value of $\operatorname{dim}(P)$ behaves monotonically as a function of $p$. Our results answer this question negatively. In particular, when $q=n^{-1 / 4}$, we have a.a.s., $\operatorname{dim}(P) \geq n-8 n^{3 / 4}$ and when $q=n^{-4 / 5}$, we have a.a.s., $\operatorname{dim}(P) \leq n-n^{4 / 5}$.

In the range $n^{-4 / 5} \leq q \leq n^{-1 / 3}$, the ratio of our two bounds on $n-\operatorname{dim}(P)$ deteriorates. In particular, for the special value of $q=n^{-1 / 2}$, we are only able to show that (roughly speaking) $n^{1 / 2} \leq n-\operatorname{dim}(P) \leq n^{3 / 4}$.

The remainder of the paper is organized as follows. In the next section, we provide essential background material. In the following three sections, we give proofs of our new bounds, grouping arguments according to the underlying scheme. The setup for the application of the inequalities of Talagrand and Janson will be given just before the results are needed. We return to the motivating extremal problems in Section 6, and we close with some brief comments on remaining problems in Section 7

\section{Essential Background Material}

For a poset $P$, we use the compact notation $a<_{P} a^{\prime}$ when $a<a^{\prime}$ in $P$. Similarly, we write $a \|_{P} a^{\prime}$ when $a$ is incomparable to $a^{\prime}$ in $P$. However, to avoid double subscripts, when $L_{j}$ is a linear extension of $P$, we will use the long form $a<a^{\prime}$ in $L_{j}$.

We will be concerned (almost exclusively) with the class $\mathbb{B}$ of bipartite posets whose ground set is the union of two disjoint antichains $A$ and $A^{\prime}$ with $A \subseteq \operatorname{Min}(P)$ and $A^{\prime} \subseteq \operatorname{Max}(P)$. Bipartite posets have been studied extensively in the literature, and we will follow here the conventions that have emerged in this research. We will write $\mathbb{B}(n)$ for the class of bipartite posets with $|A|=\left|A^{\prime}\right|=n$.

For a poset $P \in \mathbb{B}$, we let $I_{P}$ consist of all pairs $\left(a, a^{\prime}\right) \in A \times A^{\prime}$ with $a \|_{P} a^{\prime}$. Let $d \in N$, and let $\mathcal{F}=\left\{L_{1}, \ldots, L_{d}\right\}$ be a family of linear extensions of $P$. We abuse notation slightly and call $\mathcal{F}$ a realizer of $P$ if for every $\left(a, a^{\prime}\right) \in I_{P}$, there is some $j \in[d]$ with $a>a^{\prime}$ in $L_{j}$. We then define the dimension of $P$, denoted $\operatorname{dim}(P)$, as the least positive integer $d$ such that $P$ has a realizer of size $d$. It is easy to see that this altered notion of dimension never exceeds the original Dushnik-Miller 
definition, and the difference is at most 1 . In our work, an additive error of this magnitude can be safely ignored.

2.1. Matchings, Independence Number, and Clique Number. The following proposition, which holds for posets in general, is nearly self-evident. It is stated for emphasis.

Proposition 2.1. Suppose that $(x, y)$ is an ordered pair of distinct points in a poset $P$ with $x \|_{P} y$. Then there exists a linear extension $L=L(x, y)$ of $P$ such that:

(1) If $w \in P$ and $w \|_{P} x$, then $x>_{L} w$.

(2) If $z \in P$ and $z \|_{P} y$, then $z>_{L} y$.

When $P \in \mathbb{B}$ and $\left(a, a^{\prime}\right) \in A \times A^{\prime}$ with $a \|_{P} a^{\prime}$, we let $\mathcal{L}\left(a, a^{\prime}\right)$ denote the set of linear extensions of $P$ satisfying the requirements of Proposition 2.1 for the pair $\left(a, a^{\prime}\right)$.

Let $P \in \mathbb{B}$ and let $d \in[n]$. A matching (of size $d$ ) in $P$ consists of a pair $\left(T, T^{\prime}\right.$ ) of $d$-element subsets of $A$ and $A^{\prime}$, respectively, and labelings $T=\left\{a_{1}, \ldots, a_{d}\right\}$ and $T^{\prime}=\left\{a_{1}^{\prime}, \ldots, a_{d}^{\prime}\right\}$ such that $a_{j} \|_{P} a_{j}^{\prime}$ for every $j \in[d]$. There are obvious notions of maximal and maximum matchings. Also, when $T \subseteq A, T^{\prime} \subseteq A^{\prime}$, and $d=|T|=\left|T^{\prime}\right|$, we say that $T$ and $T^{\prime}$ can be matched if such labelings of $T$ and $T^{\prime}$ exist.

Lemma 2.2. Let $P \in \mathbb{B}$. Then $\operatorname{dim}(P)$ is at most the minimum size of a maximal matching in $P$.

Proof. Let $T=\left\{a_{1}, \ldots, a_{d}\right\}$ and $T^{\prime}=\left\{a_{1}^{\prime}, \ldots, a_{d}^{\prime}\right\}$ be labelings determining a matching $\left(T, T^{\prime}\right)$ of size $d$ in $P$. If this matching is maximal, we show that $\operatorname{dim}(P) \leq$ $d$. For each $j \in[d]$, let $L_{j}$ be any linear extension in $\mathcal{L}\left(a_{j}, a_{j}^{\prime}\right)$. Clearly, $\mathcal{F}=$ $\left\{L_{1}, \ldots, L_{d}\right\}$ is a realizer of $P$.

The following elementary lemma is implicit in [5] and explicit in [3].

Lemma 2.3. Let $P \in \mathbb{B}$. If $I_{P} \neq \emptyset$, and $\operatorname{dim}(P)=d$, then there is a realizer $\mathcal{F}=\left\{L_{1}, \ldots, L_{d}\right\}$ of $P$ for which there is a matching $\left(T, T^{\prime}\right)$ with $T=\left\{a_{1}, \ldots, a_{d}\right\}$ and $T^{\prime}=\left\{a_{1}^{\prime}, \ldots, a_{d}^{\prime}\right\}$ in $P$ such that $L_{j} \in \mathcal{L}\left(a_{j}, a_{j}^{\prime}\right)$ for each $j \in[d]$.

Proof. Given a realizer $\mathcal{F}=\left\{L_{1}, \ldots, L_{d}\right\}$, we note that for every $j \in[d]$, the highest element of $A$ is over the lowest element of $A^{\prime}$. If this assertion failed for some $j \in[d]$, then $\mathcal{F}-\left\{L_{j}\right\}$ would be a realizer for $P$. Now carry out the following modifications, in an iterative manner, to the linear extensions in $\mathcal{F}$. For each $j=\in[d]$, let $a_{j}$ be the highest element of $A$. Since $d=\operatorname{dim}(P)$, loss of generality, we may assume that $a_{j}>a^{\prime}$ in $L_{j}$ for every $a^{\prime} \in A^{\prime}$ with $a_{j} \|_{P} a^{\prime}$. If not, simply move all such elements from above $a_{j}$ to the gap immediately below it. An analogous remark holds for the lowest element $a_{j}^{\prime}$ of $A^{\prime}$ in $L_{j}$. Then for all $k$ with $j<k \leq d$, move $a_{j}$ to the bottom of $L_{k}$ and move $a_{j}^{\prime}$ to the top of $L_{k}$. After these steps have been taken, the resulting family is a realizer satisfying the requirements of the lemma.

Throughout this paper, we will exploit connections between posets and graphs, and we have already discussed the clique number of a graph $G$, denoted $\omega(G)$. Here is the analogous concept for bipartite posets. Let $P \in \mathbb{B}$. We call a pair $\left(V, V^{\prime}\right)$ a clique pair when $V \subseteq A, V^{\prime} \subseteq A^{\prime}$, and $v<_{P} v^{\prime}$ for all $\left(v, v^{\prime}\right) \in V \times V^{\prime}$. A clique pair $\left(V, V^{\prime}\right)$ is balanced if $\left.|V|=\mid V^{\prime}\right)$. In turn, we define the balanced clique number of $P$, denoted $\operatorname{bcn}(P)$, as the largest integer $r$ such that $P$ contains a clique pair $\left(V, V^{\prime}\right)$ 
with $|V|=\left|V^{\prime}\right|=r$. By convention, $\operatorname{bcn}(P)=0$ if there is no pair $\left(a, a^{\prime}\right) \in A \times A$ with $a<_{P} a^{\prime}$.

Let $G$ be a graph. A set $I$ of vertices in $G$ is said to be independent if there are no edges in $G$ with both endpoints in $I$. In turn, the independence number of $G$, denoted $\alpha(G)$, is the maximum size of an independent set of vertices in $G$. Analogously, when $P \in \mathbb{B}$, we will refer to a pair $\left(U, U^{\prime}\right)$ as an independent pair, when $U \subseteq A, U^{\prime} \subseteq A^{\prime}$, and $u \|_{P} u^{\prime}$ for all $\left(u, u^{\prime}\right) \in U \times U^{\prime}$. An independent pair $\left(U, U^{\prime}\right)$ is balanced if $|U|=\left|U^{\prime}\right|$, and the balanced independence number of $P$, denoted $\operatorname{bin}(P)$, is the largest integer $s$ such that $P$ contains an independent pair $\left(U, U^{\prime}\right)$ with $|U|=\left|U^{\prime}\right|=s$. Now $\operatorname{bin}(P)=0$ if there is no pair $\left(a, a^{\prime}\right) \in A \times A^{\prime}$ with $a \|_{P} a^{\prime}$.

The following lemma is implicit in 3 .

Lemma 2.4. Let $P \in \mathbb{B}$ and suppose that $\operatorname{bin}(P)<2$. If $I_{P} \neq \emptyset$, then $\operatorname{dim}(P)$ is the minimum size of a maximal matching in $P$.

Proof. Let $d=\operatorname{dim}(P)$. We know from Lemma 2.3 that $d$ is at most the minimum size of a maximal matching. We now show that this inequality is tight. Let $\mathcal{F}=$ $\left\{L_{1}, \ldots, L_{d}\right\}$ be a realizer of $P$ satisfying the requirements of Lemma 2.3. Then let $T=\left\{a_{1}, \ldots, a_{d}\right\}$ and $T^{\prime}=\left\{a_{1}^{\prime}, \ldots, a_{d}^{\prime}\right\}$ be the matching associated with $\mathcal{F}$. Set $M=A-T$ and $M^{\prime}=A^{\prime}-T^{\prime}$.

We claim that the matching evidenced by $T$ and $T^{\prime}$ is maximal. Suppose this assertion fails and there is a pair $\left(x, x^{\prime}\right) \in M \times M^{\prime}$ with $x \|_{P} x^{\prime}$. Since $\mathcal{F}$ is a realizer, there is some $j \in[d]$ with $x>x^{\prime}$ in $L_{j}$. This implies that both elements of $U=\left\{a_{j}, x\right\}$ are incomparable with both elements of $U^{\prime}=\left\{a_{j}^{\prime}, x^{\prime}\right\}$. In turn, this implies that $\left(U, U^{\prime}\right)$ is a balanced independent pair in $P$, so that $\operatorname{bin}(P) \geq 2$. The contradiction completes the proof.

\section{Matchings, Clique Number, Independence Number and Talagrand's INEQUALITY}

In this section, we prove New Upper Bound (1) and New Lower Bound (1). The proofs have the same flavor, and where their ranges overlap, we are able to determine a.a.s., the expected value of $n-\operatorname{dim}(P)$ to within a multiplicative ratio that is at most $1+o(1)$.

The arguments for these bounds require preliminary lemmas, some of which may be of independent interest. Let $s \in[n]$. Also let $S$ and $S^{\prime}$ be $s$-element subsets of $A$ and $A^{\prime}$, respectively, with $s=|S|=\left|S^{\prime}\right|$. We say the defect of the pair $\left(S, S^{\prime}\right)$ is $s$ if $\left(S, S^{\prime}\right)$ is a clique; otherwise, the defect of $\left(S, S^{\prime}\right)$ is the least non-negative integer $\delta$ such that there are subsets $T \subseteq S$ and $T^{\prime} \subseteq S^{\prime}$ with $s-\delta=|T|=\left|T^{\prime}\right|$ such that $T$ and $T^{\prime}$ can be matched.

Lemma 3.1. Suppose that $n^{-1} \log ^{2} n \leq q \leq n^{-1 / 2} \log n$. Then a.a.s., the following statement holds: If $n / 2 \leq s \leq n, S \subseteq A, S^{\prime} \subseteq A^{\prime}$, and $s=|S|=\left|S^{\prime}\right|$, then the defect of the pair $\left(S, S^{\prime}\right)$ is at most $24 / q$.

Proof. Set $\delta=24 / q$. There are at most $2^{2 n}$ pairs of the form $\left(S, S^{\prime}\right)$ where $S \subseteq A$, $S^{\prime} \subseteq A^{\prime}$ and $|S|=\left|S^{\prime}\right| \geq n / 2$. The lemma follows if we can show that for any such pair, the probability that there is no matching of size $|S|-\delta$ between $S$ and $S^{\prime}$ is $o\left(2^{-2 n}\right)$. Fix such a pair and let $s=|S|=\left|S^{\prime}\right|$.

For each non-empty subset $W \subseteq S$, let $N(W)$ consist of all elements of $S^{\prime}$ that are incomparable with at least one element of $W$. If $|N(W)| \geq|W|-\delta$, for all 
subsets $W \subseteq S$, then a matching of the desired size exists. So we consider the event $F$ that holds if there is some subset $W \subseteq S$ with $|N(W)|<|W|-\delta$.

Trivially, the inequality $|N(W)| \geq|W|-\delta$ holds when $|W| \leq \delta$. Also, if $F$ fails for all sets $W \subset A$ with $|W|=s-\delta$, then it fails for all sets $W \subset A$ with $|W|>s-\delta$. It follows that $F \subseteq \cup_{i=\delta}^{s-\delta} F_{i}$, where event $F_{i}$ holds when there is an $i$-element subset $W \subseteq S$ such that $|N(W)|<|W|-\delta$.

Now suppose that $\delta \leq i \leq s / 2$. Then there are at most $\left(\begin{array}{l}s \\ i\end{array}\right)$ choices for the set $W$. For each choice of $W$, there are at most $\left(\begin{array}{l}s \\ i\end{array}\right)$ choices for an $i$-element subset $W^{\prime}$ of $S^{\prime}$ such that $W^{\prime} \cap N(W)=\emptyset$. It follows that

$$
\begin{aligned}
\mathbb{P}\left(F_{i}\right) & \leq\left(\begin{array}{c}
s \\
i
\end{array}\right)^{2}(1-q)^{i(s-i)} & & \\
& <\exp (2 i \log n)(1-q)^{i n / 4} & & \text { since } s-i \geq n / 4 . \\
& <\exp (2 i \log n) \exp (-i q n / 4) & & \\
& <\exp (-i q n / 8) & & \text { since } q n / 4>4 \log n . \\
& <\exp (-\delta q n / 8) & & \text { since } i \geq \delta . \\
& =\exp (-3 n) & & \text { substituting for } \delta . \\
& =o\left(2^{-2 n} / n\right) . & &
\end{aligned}
$$

A symmetric calculation shows $\mathbb{P}\left(F_{i}\right)=o\left(2^{-2 n} / n\right)$ when $s / 2 \leq i \leq s-\delta$. It follows that

$$
\mathbb{P}(F) \leq \sum_{i=\delta}^{s-\delta}=o\left(2^{-2 n}\right) .
$$

This completes the proof of the lemma.

For the proof of the next lemma, we follow (essentially) the notation and terminology of Corollaries 4.3.3, 4.3.4 and 4.3.5 in Alon and Spencer [1. For a random variable $X$, we denote the expected value of $X$ as $\mathbb{E}[X]$.

Lemma 3.2. Suppose $n^{-1} \log ^{2} n \leq q \leq n^{-1 / 2} \log n$. Let $X$ be the random variable counting the number of balanced independent pairs of size 2 . Then $\mathbb{E}[X] \rightarrow \infty$ and a.a.s., $X \sim \mathbb{E}[X]$.

Proof. Set $m=\left(\begin{array}{c}n \\ 2\end{array}\right)^{2}$. Then let $\left\{\left(U_{1}, U_{1}^{\prime}\right), \ldots,\left(U_{m}, U_{m}^{\prime}\right)\right\}$ be a listing of pairs such that for each $i \in[m], U_{i}$ and $U_{i}^{\prime}$ are 2-element subsets of $A$ and $A^{\prime}$, respectively. For each $i \in[m]$, we have an event $E_{i}$ that holds if $\left(U_{i}, U_{i}^{\prime}\right)$ is a balanced independent pair. Also, we let $X_{i}$ be the associated indicator random variable. Then $X=$ $X_{1}+\cdots+X_{m}$, and we note that the random variables $X_{1}, \ldots, X_{m}$ are symmetric.

We note that since $q \geq n^{-1} \log ^{2} n$,

$$
\mathbb{E}[X]=\left(\begin{array}{l}
n \\
2
\end{array}\right)^{2} q^{4} \geq n^{4} q^{4} / 5 \geq \log ^{8} n / 5 \rightarrow \infty .
$$

We write $E_{i} \sim E_{j}$ when $i, j$ are distinct elements of $[m]$ and the events $E_{i}$ and $E_{j}$ are dependent. Clearly, $E_{i} \sim E_{j}$ when $U_{i} \times U_{i}^{\prime}$ and $U_{j} \times U_{j}^{\prime}$ intersect. When they intersect, the number of common pairs is either 1 or 2 . We fix an index $i$ and then calculate the quantity $\Delta^{*}$ defined by

$$
\Delta^{*}=\sum_{j \sim i} \mathbb{P}\left[E_{j} \mid E_{i}\right]
$$


There are $4(n-2)^{2}$ choices for the index $j$ so that $U_{i} \times U_{i}^{\prime}$ and $U_{j} \times U_{j}$ have exactly one common pair. For each such $j$, the value of $\mathbb{P}\left[E_{j} \mid E_{i}\right]$ is $q^{3}$. Similarly, there are $4(n-2)$ choices for the index $j$ so that $U_{i} \times U_{i}^{\prime}$ and $U_{j} \times U_{j}^{\prime}$ have exactly two common pairs. For each such $j$, the value of $\mathbb{P}\left[E_{j} \mid E_{i}\right]$ is $q^{2}$.

Using first that $q \geq n^{-1} \log ^{2} n$ and then that $q \leq n^{-1 / 2} \log n$, we have

$$
\Delta^{*}=4 q^{3}(n-2)^{2}+4 q^{2}(n-2)<4 q^{5} n^{4} / \log ^{4} n+4 q^{5} n^{4} / \log ^{6} n=o(\mathbb{E}[X]) .
$$

Now the conditions of Corollary 4.3.5 from [1] are satisfied and we conclude that almost always, $X \sim \mathbb{E}[X]$.

The next lemma is a straightforward application of Markov's inequality.

Lemma 3.3. Suppose $n^{-1} \log ^{2} n \leq q \leq n^{-1 / 2} \log n$ and $0<\epsilon<1$. Then a.a.s.,

$$
\operatorname{bcn}(P)<(2+\epsilon) \log (q n) / q \text {. }
$$

Proof. Set $r=(2+\epsilon) \log (q n) / q$ and let $X$ count the number of balanced clique pairs of size $r$ in $P$. Then

$$
\mathbb{E}[X]=\left(\begin{array}{l}
n \\
r
\end{array}\right)^{2}(1-q)^{r^{2}}<n^{2 r} \exp \left(-q r^{2}\right)=\exp (-2 \epsilon \log (q n))=o(1) .
$$

Since $\mathbb{E}[X]=o(1)$, it follows that a.a.s., $\operatorname{bcn}(P)<r$.

The elementary inequality in Lemma 3.3 is essentially best possible. However, this assertion is considerably more challenging to prove? 2 .

There are several different forms of Talagrand's inequalities in the literature. We will use the version given in [10, Theorem 2.29]. Let $\mathbf{R}$ and $\mathbf{R}^{+}$denote, respectively, the set of real numbers and the set of positive real numbers. When $\left(\Lambda_{1}, \ldots, \Lambda_{n}\right)$ is a sequence of subsets of $\mathbf{R}$, we denote by $\Lambda$ the product $\Lambda_{1} \times \cdots \times \Lambda_{n}$. When $i \in[n], \bar{z} \in \Lambda$, we denote by $\bar{z}(i)$ the value of coordinate $i$ of $\bar{z}$.

Theorem 3.4 (Talagrand Inequality). Let $X=f\left(Z_{1}, \ldots, Z_{n}\right)$ be a random variable determined by $n$ independent trials $Z_{1}, \ldots, Z_{n}$, where $f: \Lambda \rightarrow \mathbf{R}$ and each $Z_{i}$ takes on values in a finite set $\Lambda_{i}$. Suppose $c_{1}, \ldots, c_{n} \in \mathbf{R}^{+}$and $\psi: \mathbf{R} \rightarrow \mathbf{R}$. If for all $\bar{z}, \bar{w} \in \Lambda$, both

(1) for all $i \in[n]$, if $\bar{z}(j)=\bar{w}(j)$ for all $j \in[n]-\{i\}$, then $|f(\bar{z})-f(\bar{w})| \leq c_{i}$, and

(2) for all $\alpha \in \mathbb{R}$, if $f(\bar{z}) \geq \alpha$, then there is $J \subseteq[n]$ such that both

(a) $\sum_{j \in J} c_{j}^{2} \leq \psi(\alpha)$, and

(b) if $\bar{z}(j)=\bar{w}(j)$ for all $j \in J$, then $f(\bar{w}) \geq \alpha$,

then for every $\gamma \in \mathbb{R}$ and every $\beta \geq 0$,

$$
\mathbb{P}(X \leq \gamma-\beta) \mathbb{P}(X \geq \gamma) \leq e^{-\frac{\beta^{2}}{4 \psi(\gamma)}} .
$$

Lemma 3.5. If $n^{-1} \log ^{2} n \leq q \leq n^{-1 / 2} \log n$ and $0<\epsilon<1$, then a.a.s., $\operatorname{bcn}(P) \geq$ $(2-\epsilon) \log (q n) / q$.

Proof. Before we begin calculations, we explain how Theorem 3.4 will be applied. Label the elements of $A$ and $A^{\prime}$ arbitrarily (no assumptions about matchings) as $A=\left\{a_{1}, \ldots, a_{n}\right\}$ and $A^{\prime}=\left\{a_{1}^{\prime}, \ldots, a_{n}^{\prime}\right\}$. For each $i \in[n]$, let $\Lambda_{i}$ be the family of

\footnotetext{
${ }^{2}$ We are grateful to Tomasz Łuczak who greatly assisted us in this effort, especially the use of Talagrand's Inequality, and the setup using a vertex martingale.
} 
all subsets of $A^{\prime}$. Then it is natural to view $\Lambda$ as just a coding of the posets in $\mathbb{B}_{n}$, i.e., a poset $P \in \mathbb{B}(n)$ uniquely determines for each $i \in[n]$ the set of all $a^{\prime} \in A^{\prime}$ with $a_{i}<_{P} a^{\prime}$. In turn, the random variables $Z_{i}$ with $i \in[n]$ then capture the space $\Omega(n, p)$.

We define a function $f: \Lambda \rightarrow N$ by setting $f(P)=\operatorname{bcn}(P)$. Then we have a random variable $X=f(P)$. We note that if $P$ and $Q$ are posets in $\mathbb{B}(n)$, and there is some $a_{i} \in A$ such that the only differences between $P$ and $Q$ involve pairs from $\left\{a_{i}\right\} \times A^{\prime}$, then $|f(P)-f(Q)| \leq 1$, i.e., we take $c_{i}=1$ for all $i \in[n]$. Furthermore, whenever we have $f(P) \geq \alpha$, this can be certified by a set $J$ of size $\alpha$. So we simply take $\psi(\alpha)=\alpha$.

With $\epsilon$ fixed, we want to show that $\mathbb{P}(X>(2-\epsilon) \log (q n) / q)$ tends to 1 . Set $\delta=\epsilon / 2$. It is enough to prove that $\mathbb{P}(X>(2-\epsilon) \log (q n) / q) \geq 1-\delta$. Set $k=\gamma=(2-\delta) \log (q n) / q$ and $\beta=\delta \log (q n) / q$. Note that $\gamma-\beta=(2-\epsilon) \log (q n) / q$. Substituting these values into Talagrand's inequality, we obtain:

$$
\mathbb{P}(X \leq(2-\epsilon) \log (q n) / q) \mathbb{P}(X \geq k) \leq e^{-\frac{\delta^{2} \log ^{2}(q n)}{4 q^{2} k}} .
$$

Substituting for $k$ in the right hand side of the last inequality, we obtain

$$
\mathbb{P}(X \leq(2-\epsilon) \log (q n) / q) \mathbb{P}(X \geq k) \leq e^{-c k} \quad \text { where } c=\frac{\delta^{2}}{4(2-\delta)^{2}}
$$

This implies that either $(1) \mathbb{P}(X \leq(2-\epsilon) \log (q n) / q) \leq \delta$ or $(2) \mathbb{P}(X \geq k) \leq$ $e^{-c k} / \delta$. To complete the proof, we need only show that statement (2) cannot hold. This will be accomplished by showing that

$$
\mathbb{P}(X \geq k) \geq e^{-o(k)} .
$$

Now let $Y$ be the random variable counting the number of balanced clique pairs $\left(V, V^{\prime}\right)$ of size $k$. Then $X \geq k$ if and only if $Y>0$. Then from [10, Remark 3.1], we have

$$
\mathbb{P}(Y>0) \geq \frac{(\mathbb{E}[Y])^{2}}{\mathbb{E}\left[Y^{2}\right]}
$$

Accordingly, we want to show that $\mathbb{P}(Y>0) \geq e^{-o(k)}$. Working with the reciprocal, which simplifies the analysis, we then want to show that:

$$
\frac{\mathbb{E}\left[Y^{2}\right]}{(\mathbb{E}[Y])^{2}} \leq e^{o(k)}
$$

We have:

$$
\begin{aligned}
\frac{\mathbb{E}\left[Y^{2}\right]}{(\mathbb{E}[Y])^{2}} & =\frac{(\mathbb{E}[Y]) \sum_{i=0}^{k} \sum_{j=0}^{k}\left(\begin{array}{c}
k \\
i
\end{array}\right)\left(\begin{array}{c}
n-k \\
k-i
\end{array}\right)\left(\begin{array}{c}
k \\
j
\end{array}\right)\left(\begin{array}{c}
n-k \\
k-j
\end{array}\right)(1-q)^{k^{2}-i j}}{(\mathbb{E}[Y])^{2}} \\
& =\sum_{i=0}^{k} \sum_{j=0}^{k} \frac{\left(\begin{array}{c}
k \\
i
\end{array}\right)\left(\begin{array}{c}
n-k \\
k-i
\end{array}\right)\left(\begin{array}{c}
k \\
j
\end{array}\right)\left(\begin{array}{c}
n-k \\
k-j
\end{array}\right)}{\left(\begin{array}{c}
n \\
k
\end{array}\right)^{2}}(1-q)^{-i j} .
\end{aligned}
$$

There are $(k+1)^{2}$ terms in the sum, so it suffices to show that every term has size at most $\exp (o(k))$. Clearly, this holds whenever $i=0$ or $j=0$. So we are concerned only with terms where $i, j \geq 1$. Trivially, we have $\left(\begin{array}{c}k \\ i\end{array}\right)\left(\begin{array}{l}n-k \\ k-i\end{array}\right) \leq\left(\begin{array}{l}n \\ k\end{array}\right)$, so that

$$
\frac{\left(\begin{array}{c}
k \\
i
\end{array}\right)\left(\begin{array}{l}
n-k \\
k-i
\end{array}\right)}{\left(\begin{array}{l}
n \\
k
\end{array}\right)} \leq 1
$$


A symmetric inequality holds for $j$.

Using only the elementary bound in (5), we observe that if $i \leq k / \log ^{2}(q n)$, then the term for the pair $(i, j)$ is at most

$$
\begin{aligned}
(1-q)^{-i j} & \leq \exp \left(q k^{2} / \log ^{2}(q n)\right) \\
& \leq \exp \left(q k \frac{2 \log (q n)}{q} / \log ^{2}(q n)\right) \\
& =\exp (2 k / \log (q n)) \\
& =\exp (o(k))
\end{aligned}
$$

A symmetric statement holds when $j \leq k / \log ^{2}(q n)$.

Now we focus on the terms when $i, j \geq k / \log ^{2}(q n)$. For such terms, we have the following improved bound.

$$
\frac{\left(\begin{array}{c}
k \\
i
\end{array}\right)\left(\begin{array}{c}
n-k \\
k-i
\end{array}\right)}{\left(\begin{array}{l}
n \\
k
\end{array}\right)}<\left(\begin{array}{c}
k \\
i
\end{array}\right) \frac{\left(\begin{array}{c}
n \\
k-i
\end{array}\right)}{\left(\begin{array}{c}
n \\
k
\end{array}\right)}<\left(\frac{3 k}{i}\right)^{i}\left(\frac{k}{n}\right)^{i}=\left(\frac{3 k^{2}}{i n}\right)^{i} .
$$

A symmetric inequality holds for $j$. Accordingly, when $k / \log ^{2}(q n) \leq i, j \leq k$, we have the following upper bound on the term for $(i, j)$ :

$$
\left(\frac{3 k \log ^{2}(q n)}{n}\right)^{i+j} \exp (q i j)
$$

We assume without loss of generality that $i \leq j$. We then take the logarithm of the expression in (6) to obtain:

$$
(i+j)[\log 3+\log k+2 \log \log (q n)-\log n]+q i j
$$

Considering $j$ fixed, this is a linear function of $i$, defined on the interval $\left[k / \log ^{2}(q n), j\right]$. So it achieves its maximum value either at $i=k / \log ^{2}(q n)$ or at $i=j$. The choice depends on the sign of the coefficient of $i$, which is

$$
\begin{aligned}
& \log 3+\log k+2 \log \log (q n)-\log n+q j= \\
& \log 3+\log (2-\delta)+3 \log \log (q n)-\log (q n)+q j .
\end{aligned}
$$

Case 1. $j \leq(\log (q n)-3 \log \log (q n)-\log 3-\log (2-\delta)) / q$.

In this case, the coefficient of $i$ is negative, so the maximum value is achieved when $i=k / \log ^{2}(q n)$. The term associated with $(i, j)$ is less than $\exp (q i j)$, and for $j$, we use the generous upper bound bound $j \leq \log (q n) / q$. It follows that the term for $(i, j)$ is at most:

$$
\exp \left(q \frac{k}{\log ^{2}(q n)} \frac{\log (q n)}{q}=\exp (k / \log (q n)=\exp (o(k)) .\right.
$$

Case 2. $j>(\log (q n)-3 \log \log (q n)-\log 3-\log (2-\delta)) / 2$.

In this case, the maximum value is achieved when $i=j$, and the term associated with $(i, j)$ is at most:

$$
\left(\left(\frac{9 k^{2} \log ^{4}(q n)}{n^{2}}\right)^{2} \exp (q j)\right)^{j}
$$


In (9), we note that

$$
\exp (q j) \leq \exp (q k)=(q n)^{2-\delta} .
$$

Using this inequality and substituting for $k$, the expression in (9) becomes:

$$
\left(\frac{9 \log ^{6}(q n)}{(q n)^{\delta}}\right)=o(1)
$$

With this observation, the proof of the lemma is complete.

Lemma 3.6 (New Upper Bound (1)). Suppose $n^{-1} \log ^{2} n \leq q \leq n^{-1 / 2} \log n$ and $0<\epsilon<1$. Then a.a.s., $\operatorname{dim}(P) \leq n-(2-\epsilon) \log (q n) / q$.

Proof. Let $\epsilon_{1}=\epsilon / 2$. Then set $r=\left(2-\epsilon_{1}\right) \log (q n) / q$. Using Lemma 3.5, it follows that a.a.s., the following two statements hold: (1) the balanced clique number of $P$ is at least $r$, and (2) for every pair $\left(S, S^{\prime}\right)$, with $S \subseteq A, S^{\prime} \subseteq A^{\prime}$ and $|S|=\left|S^{\prime}\right| \geq n / 2$, the defect of $\left(S, S^{\prime}\right)$ is less than $24 / q$.

Set $\delta=24 / q$, and let $\left(V, V^{\prime}\right)$ be a balanced clique pair of size $r$ in $P$. Set $S=A-V$ and $S^{\prime}=A^{\prime}-V^{\prime}$. Note that $|S|=|S|>n / 2$. Let $\left(T, T^{\prime}\right)$ be a maximum matching in $S \cup S^{\prime}$. Then $|T|=\left|T^{\prime}\right| \geq|S|-\delta$. Let $Q$ be the subposet of $P$ determined by the points in the matching together with the points in $V \cup V^{\prime}$. Then $\left(T, T^{\prime}\right)$ is a maximal matching in $Q$. It follows from Lemma 2.2 that $\operatorname{dim}(Q) \leq|T| \leq n-r$. We note that

$$
2 \delta<48 / q<\epsilon_{1} \log (q n) / q .
$$

The removal of a point from a bipartite poset decreases dimension by at most 1 , and $Q$ is obtained from $P$ by removing at most $2 \delta$ points. It follows that a.a.s.,

$$
\operatorname{dim}(P) \leq \operatorname{dim}(Q)+2 \delta \leq(n-r)+\epsilon_{1} \log (q n) / q=n-(2-\epsilon) \log (q n) / q .
$$

Readers will note that the proof of the next result uses the "alteration" method (see Chapter 3 in Alon and Spencer [1]) first used by Erdős in his probabilistic proof of the existence of graphs with large girth and large chromatic number.

Lemma 3.7 (New Lower Bound (1)). Suppose $0<\epsilon<1$. If $n^{-1} \log ^{2} n \leq q \leq$ $n^{-4 / 5}$, then a.a.s., $\operatorname{dim}(P)>n-(2+\epsilon) \log (q n) / q$.

Proof. Set $\epsilon_{1}=\epsilon / 2$ and $r=\left(2+\epsilon_{1}\right) \log (q n) / q$. Using Lemma 3.3, we know that a.a.s., the balanced clique number of $P$ is less than $r$.

Let $X$ be the random variable counting the number of balanced independent pairs $\left(U, U^{\prime}\right)$ with $|U|=\left|U^{\prime}\right|=2$. Using Lemma 3.2 we know that a.a.s., $X \sim E[X]$. Since $E[X]=\left(\begin{array}{c}n \\ 2\end{array}\right)^{2} q^{4} \sim q^{4} n^{4} / 4$, we will settle for the weaker inequality a.a.s., $X \leq n^{4} q^{4} / 2$. When $q \leq n^{-4 / 5}$, this implies that a.a.s., $X<\epsilon_{1} \log (q n) / q$.

It follows that there are subsets $S \subset A$ and $S^{\prime} \subset A^{\prime}$ with $|S|=\left|S^{\prime}\right|=n-$ $\epsilon_{1} q^{-1} \log (q n)$ such that a.a.s., the subposet $Q$ of $P$ with ground set $S \cup S^{\prime}$ has balanced clique number less than $r$ and balanced independence number less than 2 Then a.a.s.,

$$
\begin{aligned}
\operatorname{dim}(P) & \geq \operatorname{dim}(Q) \\
& >\left(n-\epsilon_{1} \log (q n) / q\right)-\left(2+\epsilon_{1}\right) \log (q n) / q \\
& =n-(2+\epsilon) \log (q n) / q .
\end{aligned}
$$




\section{Lower Bounds and an Application of Janson's Inequality}

In this section, we prove New Lower Bounds (2), (3) and (4). All three proofs require concepts developed in [5], starting with a good bound on the expected value of the balanced independence number. The following elementary lemma uses only Markov's inequality.

Lemma 4.1. Suppose $n^{-1} \log ^{2} n / n \leq q \leq 1 / 2$ and $0<\epsilon<1$. Then a.a.s.,

$$
\operatorname{bin}(P)<t:=\lceil 2[\log n+\log \log (1 / q)] / \log (1 / q)\rceil .
$$

Proof. Let $Y$ count the number of balanced independent pairs of size $t$. Then

$$
\mathbb{E}[Y]=\left(\begin{array}{l}
n \\
t
\end{array}\right)^{2} q^{t^{2}}<\exp (t(2 \log n-t \log (1 / q)))=o(1) .
$$

With this lemma in mind, for the balance of this section, whenever the value of $q$ is specified, we set:

$$
t=\lceil(2 \log n+\log \log n) / \log (1 / q)\rceil .
$$

Fix a value of $q$, with $t$ then determined by (10). A short pair is a pair $\left(\sigma, \sigma^{\prime}\right)$ where $\sigma$ is a linear order on a $(t-1)$-element subset of $A$, and $\sigma^{\prime}$ is a linear order on a $(t-1)$-element subset of $A^{\prime}$. Let $d \in[n]$ and let $\Sigma=\left\{\left(\sigma_{j}, \sigma_{j}^{\prime}\right): 1 \leq j \leq d\right\}$ be a family of short pairs. With the family $\Sigma$ fixed, we make the following definitions.

For a pair $\left(a, a^{\prime}\right) \in A \times A^{\prime}$ and an integer $j \in[d]$,

(1) Event $R_{j}\left(a, a^{\prime}\right)$ holds if $a \in \sigma_{j}$ and $b \|_{P} a^{\prime}$ for all $b \in \sigma_{j}$ with $h_{j}(b)<h_{j}(a)$.

(2) Event $R_{j}^{\prime}\left(a, a^{\prime}\right)$ holds if $a^{\prime} \in \sigma_{j}^{\prime}$ and $b^{\prime} \|_{P} a$ for all $b^{\prime} \in \sigma_{j}^{\prime}$ with $h_{j}^{\prime}\left(b^{\prime}\right)<$ $h_{j}^{\prime}\left(a^{\prime}\right)$.

We note that $R_{j}\left(a, a^{\prime}\right)$ holds whenever $a$ is the highest element of $\sigma_{j}$. Also, $R_{j}^{\prime}\left(a, a^{\prime}\right)$ holds whenever $a^{\prime}$ is the lowest element of $\sigma_{j}^{\prime}$.

For a pair $\left(a, a^{\prime}\right) \in A \times A^{\prime}$, let $\left(a<a^{\prime}\right)$ be the event that holds when $a<_{P} a^{\prime}$. Also, let $\left(a \| a^{\prime}\right)$ be the event that holds when $a \|_{P} a^{\prime}$. Now set

$$
R\left(a, a^{\prime}\right)=\left(a<a^{\prime}\right) \vee\left(\vee_{j \in[d]} R_{j}\left(a, a^{\prime}\right)\right) \vee\left(\vee_{j \in[d]} R_{j}^{\prime}\left(a, a^{\prime}\right)\right) .
$$

We say that $\Sigma$ realizes the pair $\left(a, a^{\prime}\right)$ when $R\left(a, a^{\prime}\right)$ holds

In turn set

$$
R(P)=\wedge_{\left(a, a^{\prime}\right) \in A \times A^{\prime}} R\left(a, a^{\prime}\right) .
$$

We say $\Sigma$ is a short realizer for $P$ when $R(P)$ holds. Then we define the short dimension of $P$, denoted $\operatorname{sdim}(P)$, as the least positive integer $d$ such that there is a family $\Sigma=\left\{\left(\sigma_{j}, \sigma_{j}^{\prime}\right): j \in[d]\right\}$ of short pairs such that $\Sigma$ is a short realizer of $P$.

We observe that a.a.s., $\operatorname{sdim}(P) \leq \operatorname{dim}(P)$. To see this, let $\mathcal{F}=\left\{L_{1}, \ldots, L_{d}\right\}$ be a realizer of $P$. Then for each $j \in[d]$, let $\sigma_{j}$ be the linear order consisting of the highest $t-1$ elements of $A$ in $L_{j}$. Also, let $\sigma_{j}^{\prime}$ be the linear order consisting of the lowest $t-1$ elements of $A^{\prime}$ in $L_{j}$. Since a.a.s., the balanced independence number of $P$ is less than $t$, it follows that a.a.s., $\Sigma=\left\{\left(\sigma_{j}, \sigma_{j}^{\prime}\right): j \in[d]\right\}$ is a short realizer for $P$. Accordingly, a lower bound on $\operatorname{sdim}(P)$ is also a lower bound on $\operatorname{dim}(P)$.

The next step in the argument for all three lower bounds is to fix a short family $\Sigma=\left\{\left(\sigma_{j}, \sigma_{j}^{\prime}\right): 1 \leq j \leq d\right\}$ and consider the event $R(P)$ that holds when $\Sigma$ is a short realizer for $P$. We will determine a reasonably accurate upper bound $p_{0}$ on $\mathbb{P}(R(P))$. The number of short families is less than $n^{2(t-1) d}$ and $d \leq n$, so we can say that a.a.s., $\operatorname{sdim}(P)>d$ if $n^{2 t n} p_{0}=e^{2 t n \log n} p_{0}=o(1)$. 
With $\Sigma$ fixed, let $T$ consist of those elements $a \in A$ such that there is at least one $j \in[d]$ with $a$ the highest element of $A$ in $\sigma_{j}$. Then set $M=A-T$. Analogously, let $T^{\prime}$ consist of those elements $a^{\prime} \in A^{\prime}$ such that there is at least one $j \in[d]$ with $a^{\prime}$ the lowest element of $\sigma_{j}^{\prime}$. Then set $M^{\prime}=A^{\prime}-T^{\prime}$. Also, set $s=t-2$.

We note that $R\left(a, a^{\prime}\right)$ holds whenever $a \in T$ or $a^{\prime} \in T^{\prime}$. Accordingly,

$$
R(P)=\wedge_{\left(x, x^{\prime}\right) \in M \times M^{\prime}} R\left(x, x^{\prime}\right) .
$$

Our next goal will be to determine a bound on $d$ that forces $\mathbb{P}(R(P))$ to be exponentially small. Some additional notation and terminology is required. We describe this notation in full detail for $M$. The notation for $M^{\prime}$ is dual.

When $j \in[d], x \in M$, and $x \in \sigma_{j}$, we let $h_{j}(x)$ count the number of elements $y \in \sigma_{j}$ with $y$ higher than $x$ in $\sigma_{j}$. By convention, we set $h_{j}(x)=\infty$ if $x \notin \sigma_{j}$. It is natural to view the quantity $h_{j}(x)$ as the height of $x$ in $\sigma_{j}$. For an integer $i \in[s]$, we then let $\mu_{i}(x)$ count the number of $j \in[d]$ with $h_{j}(x / W)=i$. We view $\mu_{i}(x)$ as the multiplicity of $x$ for height $i$. Then define the quantity $w(x)$ by setting

$$
w(x)=\sum_{i=1}^{s} \mu_{i}(x) 2^{1-i} .
$$

We view the quantity $w(x)$ as the weight of $x$. Note that

$$
\sum_{x \in M} w(x)<2 d .
$$

Since $d \leq n$, there is a subset $M_{0} \subset M$ with $\left|M_{0}\right|=m / 2$ such that $w(x)<4 d / m$ for every $x \in M_{0}$.

The preceding discussion is followed in a dual manner to determine a subset $M_{0}^{\prime} \subset M^{\prime}$ with $\left|M_{0}^{\prime}\right|=m / 2$ so that $w\left(x^{\prime}\right)<4 d / m$ for every $x^{\prime} \in M_{0}^{\prime}$. Set $\mathcal{I}=$ $M_{0} \times M_{0}^{\prime}$. In the analysis to follow, we will need the following elementary fact. It is stated formally, as we will need it again in the following section.

Proposition 4.2 (Weight-Shift). If $0 \leq q \leq 1 / 2$ and $i \in N$, then $1-q^{i}<$ $\left(1-q^{i+1}\right)^{2}$.

For an event $E$ in a probability space $\Omega$, we use the notation $\bar{E}$ to denote the event that holds when $E$ fails. Let $\left(x, x^{\prime}\right) \in \mathcal{I}$. We consider the events in the family $\left\{\bar{R}_{j}\left(x, x^{\prime}\right): j \in[d]\right\}$. As explained in [5, these events are positively correlated, i.e., when $j$ and $k$ are distinct integers in $[d]$,

$$
\left.\mathbb{P}\left(\bar{R}_{j}\left(x, x^{\prime}\right) \mid \bar{R}_{k}\left(x, x^{\prime}\right)\right) \geq \mathbb{P}\left(\bar{R}_{j}\left(x, x^{\prime}\right)\right)\right) .
$$

With the convention that $1-q^{\infty}=1$, it follows that:

$$
\begin{array}{rlrl}
\mathbb{P}\left(\wedge_{j \in[d]} \bar{R}_{j}\left(x, x^{\prime}\right)\right. & \geq \prod_{j \in[d]}\left(1-q^{h_{j}(x)}\right) & & \text { Using correlation. } \\
& \geq \prod_{i \in[s]}\left(1-q^{i}\right)^{\mu_{i}(x)} & & \text { Definition of multiplicity. } \\
& \geq(1-q)^{w(x)} & & \text { Using Proposition 4.2. } \\
& \geq(1-q)^{4 n / m} . &
\end{array}
$$

Analogously, we have:

$$
\mathbb{P}\left(\wedge_{j \in[d]} \bar{R}_{j}^{\prime}\left(x, x^{\prime}\right) \geq(1-q)^{4 n / m} .\right.
$$


It follows that

$$
\mathbb{P}\left(R\left(x, x^{\prime}\right)\right) \leq 1-q(1-q)^{8 n / m} .
$$

4.1. Applying the Janson Inequality. We will use the Janson inequality in the proofs of New Lower Bounds (2) and (3). Here is the set up for this result, following (essentially) the presentation in Chapter 8 of Alon and Spencer [1]. The text [10] by Janson, Łuczak and Rucinski is cited for the proof.

Let $\mathcal{I}$ be a finite set and let $\left\{F_{i}: i \in \mathcal{I}\right\}$ be a finite family of events in a probability space $\Omega$. When $i$ and $j$ are distinct elements of $\mathcal{I}$, we write $F_{i} \sim F_{j}$ when $F_{i}$ and $F_{j}$ are dependent. Also, we set

$$
\Delta=\sum\left\{\mathbb{P}\left(F_{i} \wedge F_{j}\right):(i, j) \in \mathcal{I} \times \mathcal{I}, F_{i} \sim F_{j}\right\},
$$

and

$$
\mu=\sum_{i \in \mathcal{I}} \mathbb{P}\left(F_{i}\right)
$$

Here is the statement of the Janson inequality we will apply.

Theorem 4.3 (Janson Inequality). Let $\left\{F_{i}: i \in \mathcal{I}\right\}$ be a finite family of events with $\mathbb{P}\left(F_{i}\right) \leq 1 / 2$ for all $i \in \mathcal{I}$. If $\Delta \leq \mu$, then

$$
\mathbb{P}\left(\wedge_{i \in \mathcal{I}} \overline{F_{i}}\right) \leq \exp (-\mu / 2) .
$$

Lemma 4.4 (New Lower Bounds (2) and (3)). If $n^{-4 / 5} \leq q \leq \frac{1}{8} n^{-1 / 4} \log ^{3} n$, then a.a.s.,

$$
\operatorname{dim}(P)> \begin{cases}n-32(n \log n / q)^{1 / 2} & \text { if } n^{-4 / 5} \leq q \leq(16)^{1 / 3} n^{-1 / 3} \log ^{1 / 3} n . \\ n-8 q n & \text { if }(16)^{1 / 3} n^{-1 / 3} \log ^{1 / 3} n \leq q \leq \frac{1}{8} n^{-1 / 4} \log ^{3} n .\end{cases}
$$

Proof. For this range, we note that $t \leq 9$. For every $\left(x, x^{\prime}\right) \in \mathcal{I}$, we have an event $F\left(x, x^{\prime}\right)$ that holds when $\Sigma$ fails to realize the pair $\left(x, x^{\prime}\right)$. Note that for $F\left(x, x^{\prime}\right)$ to hold, we need $x \|_{P} x^{\prime}$, so $\mathbb{P}\left(F\left(x, x^{\prime}\right)\right) \leq q \leq 1 / 2$. We also observe that $\mathbb{P}\left(F\left(x, x^{\prime}\right)\right) \geq q(1-q)^{8 n / m}$. Set $m=q n z$ where $z \geq 8$. In general, we will have $z \rightarrow \infty$, but the restriction $z \geq 8$ is enough to imply that $(1-q)^{8 n / m} \geq 1 / 4$. It follows that $\mu$, the exected number of pairs that fail, is at least $m^{2} q / 16$.

When $x, y \in M_{0}$ and $x^{\prime}, y^{\prime} \in M_{0}^{\prime}$, we observe that $F\left(x, x^{\prime}\right) \sim F\left(y, y^{\prime}\right)$ if and only if $\left|\left\{x, x^{\prime}\right\} \cap\left\{y, y^{\prime}\right\}\right|=1$. Furthermore, when $F\left(x, x^{\prime}\right) \sim F\left(y, y^{\prime}\right)$, and event $F\left(x, x^{\prime}\right) \wedge F\left(y, y^{\prime}\right)$ holds, we must have $x \|_{P} x^{\prime}$ and $y \|_{P} y^{\prime}$. These two events are independent and each has probability $q$. It follows that

$$
\mathbb{P}\left(F\left(x, x^{\prime}\right) \wedge F\left(y, y^{\prime}\right)\right) \leq q^{2} .
$$

There are are $2(m / 2)(m / 2)$ such pairs so $\Delta \leq m^{2} q^{2} / 2$. To apply the Janson inequality, we need $\Delta \leq \mu$, but this simply requires $q \leq 1 / 8$. We conclude that

$$
\mathbb{P}(\mathbb{R}(\Sigma)) \leq \mathbb{P}\left(\wedge_{\left(x, x^{\prime}\right) \in \mathcal{I}} \overline{F\left(x, x^{\prime}\right)}\right) \leq \exp (-\mu / 2) \leq \exp \left(-m^{2} q / 32\right) .
$$

Recall that the number of short families is less than $n^{2 t n} \leq \exp (18 n \log n)$, since $t \leq 9$. Noting that $18 \cdot 32=576<2^{10}$, can conclude that a.a.s., $\operatorname{dim}(P)>d$ if $2^{10} n \log n \leq m^{2} q$. Since $m=q n z$, this becomes $2^{10} \log n \leq q^{3} n z^{2}$. This requires

$$
z \geq 32\left(\log n /\left(q^{3} n\right)\right)^{1 / 2} \text {. }
$$


When $z=8$, this inequality holds when $q \geq(16)^{1 / 3} n^{-1 / 3} \log ^{1 / 3} n$, and this completes the proof of New Lower Bound (3).

Now we assume that $q \leq(16)^{1 / 3} n^{-1 / 3} \log ^{1 / 3} n$. Now we treat inequality (12) as an equation, i.e., we set $z=32\left(\log n /\left(q^{3} n\right)\right)^{1 / 2}$. The equation $m=q n z$ is equivalent to $m=32(n \log n / q)^{1 / 2}$, and with this observation, the proof of New Upper Bound (2) is complete.

4.2. A Family of Independent Events. To obtain a proof of New Lower Bound (4), we simply update the original argument in [5] as given on pages $262-268$. We have elected not to repeat the details of this argument. Instead, we will provide only an outline of the steps to be taken, with notational changes made to agree with our treatment here

(1) We identify a subset $\mathcal{J}$ of $M \times M^{\prime}$ with $|\mathcal{J}|=m^{3} /\left(72 n s^{2}\right)$.

(2) For each $\left(x, x^{\prime}\right) \in \mathcal{I}$, we determine an event $E\left(x, x^{\prime}\right)$ such that $R\left(x, x^{\prime}\right) \subseteq$ $E\left(x, x^{\prime}\right)$ and $\operatorname{Pr}\left(E\left(x, x^{\prime}\right)\right) \geq q(1-q)^{24 n / m}$.

(3) Events in the family $\left\{E\left(x, x^{\prime}\right):\left(x, x^{\prime}\right) \in \mathcal{J}\right\}$ are independent.

Since the events in $\mathcal{J}$ are independent, it follows that

$$
\mathbb{P}(R(P)) \leq\left[1-q(1-q)^{24 n / m}\right]^{\frac{m^{3}}{72 n s^{2}}} .
$$

Inequality (13) provides an upper bound on $p_{0}$, the maximum value of $\mathbb{P}(R(P))$. To show that a.a.s., $\operatorname{dim}(P)>d=n-m$, it suffices to require that:

$$
e^{2 t n \log n}\left[1-q(1-q)^{24 n / m}\right]^{m^{3} / 72 n s^{2}}=o(1)
$$

In the range we consider, it will always be the case that $q(1-q)^{24 n / m}=o(1)$. With this restriction, the preceding inequality holds if:

$$
n^{2} t^{3} \log n=o\left(q(1-q)^{24 n / m} m^{3}\right) .
$$

In the proof of the next lemma, we will refer to (14) as the "master inequality."

Lemma 4.5 (New Lower Bound (4)). If $\frac{1}{8} n^{-1 / 4} \log ^{3} n \leq q \leq 1 / 2$ and $z=\log n+$ $4 \log q-8 \log \log n$, then a.a.s., $\operatorname{dim}(P) \geq n-24 q n / z$.

Proof. Set $m=24 q n / z$. The lower bound on $q$ implies $q n^{4} \geq \log ^{11} n$. It follows that $z \geq 3 \log \log n$ so that $m=o(q n)$. With this value of $m$, we can safely approximate $(1-q)^{24 n / m}$ as $e^{-z}$. Accordingly, the master inequality becomes $n^{2} t^{3} \log n=o\left(q e^{-z} q^{3} n^{3} / z^{3}\right)$, which is equivalent to $t^{3} z^{3} e^{z} \log n=o\left(q^{4} n\right)$. We note that $e^{z}=q n^{4} / \log ^{8} n$. So the master inequality holds if $t^{3} z^{3} \log n=o\left(\log ^{8} n\right)$. However, $t \leq 3 \log n$. Furthermore, $z<\log n$. It follows that $t^{3} z^{3} \log n=O\left(\log ^{7} n\right)$, so that the master inequality holds.

We observe that there is a threshold occurring when $q \sim n^{-1 / 4}$. When $q$ is below this threshold, the Janson inequality approach gives a better result, and when $q$ is above this threshold, the original approach using a family of independent events is better. 


\section{Generalized Latin Rectangles and the Euler Product Function}

In this section, we prove New Upper Bounds (2) and (3). The arguments require a "one-sided" reformulation of dimension, using the same approach (at least one half of it) taken in the last section. Let $P \in \mathbb{B}$ and let $\mathcal{F}=\left\{\sigma_{1}, \ldots, \sigma_{d}\right\}$ be a nonempty family of linear orders such that for each $j \in[d], \sigma_{j}$ is a linear order on a non-empty subset of $A$. Now there is no restriction on the size of these linear orders. For a pair $\left(a, a^{\prime}\right) \in A \times A^{\prime}$, we say that $\mathcal{F}$ realizes $\left(a, a^{\prime}\right)$ if either $(1) a<_{P} a^{\prime}$ or (2) there is some $j \in[d]$ with $a \in \sigma_{j}$ and $b \|_{P} a^{\prime}$ for all $b^{\prime} \in \sigma_{j}$ with $h_{j}(b)<h_{j}(a)$. In turn, we say $\mathcal{F}$ is a one-sided realizer for $P$ when $\mathcal{F}$ realizes $\left(a, a^{\prime}\right)$ for all pairs $\left(a, a^{\prime}\right) \in A \times A^{\prime}$.

Clearly, $\operatorname{dim}(P)$ is the least $d \geq 1$ for which $P$ has a one-sided realizer of size $d$. Our strategy for proving New Upper Bounds (1) and (2) will be to design a single candidate family $\mathcal{F}=\left\{\sigma_{1}, \ldots, \sigma_{d}\right\}$ and show that a.a.s., this family is a one-sided realizer of a poset $P$. To implement this strategy, we must pause to establish a connection with a classic concept in combinatorics.

5.1. Generalized Latin Rectangles. Recall that when $m$ and $s$ are integers with $1 \leq s \leq m$, an $s \times m$ array (matrix) $R$ is called a latin rectangle when (1) each row of $R$ is a permutation of the integers in $[m]$, and $(2)$ the entries in each column of $R$ are distinct. As is well known, if $2 \leq s \leq m$, an $(s-1) \times m$ latin rectangle $R$ can always be extended to an $s \times m$ latin rectangle by adding a new row.

Now let $(m, r, s)$ be a triple of positive integers. An $s \times(r m)$ array $R$ of integers from $[m]$ will be called an $(m, r, s)$ - GLR (where GLR is an abbreviation for generalized latin rectangle) when the following conditions are met:

(1) In each row of $R$, each integer in $[m]$ occurs exactly $r$ times.

(2) In each column $C$ of $R$, the $s$ integers occuring in column $C$ are distinct.

(3) For each distinct pair $i, j \in\{1,2, \ldots, m\}$, there is at most one column $C$ in $R$ for which $i$ is below $j$ in column $C$.

Note that when $r=1$, the third requirement is not part of the traditional definition for a latin rectangle. However, it will be soon be clear why we want this additional restriction in place.

Here is an example of a $(9,2,3)$-GLR.

$$
\left[\begin{array}{llllllllllllllllll}
1 & 1 & 2 & 2 & 3 & 3 & 4 & 4 & 5 & 5 & 6 & 6 & 7 & 7 & 8 & 8 & 9 & 9 \\
8 & 9 & 9 & 1 & 1 & 2 & 2 & 3 & 3 & 4 & 4 & 5 & 5 & 6 & 6 & 7 & 7 & 8 \\
3 & 6 & 4 & 7 & 5 & 8 & 6 & 9 & 7 & 1 & 8 & 2 & 9 & 3 & 1 & 4 & 2 & 5
\end{array}\right]
$$

The reader may note that it is impossible to extend this array to a $(9,2,4)-$ GLR. More generally, we have the following natural extremal problem: For a pair $(m, r)$ of positive integers, find the the largest integer $s=f(m, r)$ for which there is an $(m, r, s)-$ GLR. Trivially, $f(m, r) \geq 1$.

Lemma 5.1. Let $m, r, s$ be positive integers with $s \geq 2$. If $f(m, r) \geq s$, then $r s(s-1) \leq 2(m-1)$.

Proof. Suppose that $R$ is an $(m, r, s)-$ GLR. There are $r m$ columns in $R$ and for each column $C$ in $R$, there are $s(s-1) / 2$ ordered pairs $(i, j)$ where $i$ is below $j$ in column $C$. The last two conditions in the definition of an $(m, r, s)$-GLR force $r m s(s-1) / 2 \leq m(m-1)$, so that $r s(s-1) \leq 2(m-1)$. 
Lower bounds on this extremal problem are more challenging, but we will give an explicit construction which is sufficient for our purposes. If $R$ is an $(m, r, s)-$ GLR, we say $R$ is resolvable if it consists of $r$ latin rectangles placed side by side, i.e., each row partitions into $r$ blocks of consecutive elements and each block is a permutation of $[m]$. The following lemma is an elementary extension of the classical result for latin rectangles, and we only outline the proof, leaving the details as an exercise for students.

Lemma 5.2. Let $m, r$ and $s$ be positive integers with $s \geq 2$. If $m>2 r s^{3}$, then there is an $(m, r, s)-$ GLR.

Proof. Consider the problem of adding a last row to a resolvable $(m, r, s-1)-$ GLR. Proceeding block by block, we have a balanced $(m, m)$ bipartite graph $G$ with positions 1 through $m$ on one side of $G$ and sets of allowable choices for each of the $m$ positions on the other side. Clearly, the most challenging case in completing the last row is the last block.

Consider one of the $m$ columns in the last block, and let $x$ be one of the $s-1$ integers that already occurs in this column. Then $x$ is over $(r-1) s(s-1) / 2$ other integers in the first $r-1$ blocks, and $x$ is over $(s-1)(s-2) / 2$ other integers in the last block. When $x$ is the lowest element in the column, then $x$ itself is not allowable. It follows that the number of allowable choices is:

$$
m-(r-1) s(s-1)^{2} / 2-(s-1)^{2}(s-2) / 2-1
$$

Note that the inequality $2 r s^{3}<m$ implies that the quantity in inequality (15) is at least $m / 2$. A parallel argument shows that each of the integers in $[\mathrm{m}]$ belongs to at least $m / 2$ of the sets of allowable choices. It is an immediate consequence of Hall's theorem that a balanced bipartite graph $G$ with $2 m$ vertices and minimum degree $\delta(G) \geq m / 2$ has a complete matching. Futhermore, a matching in $G$ provides a legal way to complete the last row.

Let $m, r, s$ be integers, and let $R$ be an $(m, r, s)-$ GLR. We set $d=m r$ and $n=d+m$. We fix an arbitrary $d$-element subset $T=\left\{a_{1}, \ldots, a_{d}\right\}$ of $A$ and set $M=A-T=\left\{x_{1}, \ldots, x_{m}\right\}$. We construct a family $\mathcal{F}=\left\{\sigma_{1}, \ldots, \sigma_{d}\right\}$ of linear orders on $(s+1)$-element subsets of $A$ as follows. For each $j \in[d]$, we set $h_{j}\left(a_{j}\right)=0$. Then for each pair $(i, j) \in[s] \times[d]$, we set $h_{j}\left(x_{\alpha}\right)=i$ when the integer in row $i$ and column $j$ of $R$ is $\alpha$.

When $\left(a, a^{\prime}\right) \in A \times A^{\prime}$, it is obvious that $\mathcal{F}$ realizes $\left(a, a^{\prime}\right)$ if $a \in T$. Let $X$ be the random variable counting the number of pairs $\left(a, a^{\prime}\right) \in A \times A^{\prime}$ for which $\mathcal{F}$ fails to realize $\left(a, a^{\prime}\right)$. Since $a$ must belong to $M$, the expected value of $X$ is given by:

$$
E[X]=n m q\left[\prod_{i=1}^{s}\left(1-q^{i}\right)\right]^{r} .
$$

Note that the expression $\prod_{i=1}^{s}\left(1-q^{i}\right)$ is a partial product of the Euler product function $\phi(q)=\prod_{i=1}^{\infty}\left(1-q^{i}\right)$. Using the weight-shift propososition 4.2 from the preceding section, it follows that

$$
\left(1-q^{s}\right) \prod_{i=1}^{s}\left(1-q^{i}\right)<\phi(q)<\prod_{i=1}^{s}\left(1-q^{i}\right) .
$$


Lemma 5.3 (New Upper Bound (3)). If $n^{-1 / 3} \leq q \leq 1 / 2$ and $z=n^{2} q \log (1 / \phi(q))$, then a.a.s., $\operatorname{dim}(P)<n-n \log (1 / \phi(q)) / \log z$.

Proof. We will only consider values of $m=n-d$ with $m \geq q n / \log ^{10} n$. With this restriction $r=d / m=n / m-1 \leq \log ^{11} n / q$. The requirement for the existence of a $(m, r, s)-$ GLR is $m>2 r s^{3}$. With the restrictions on $q$ and $m$, the requirement is met when $s=n^{1 / 10}$. For such a large value of $s$, we are safe if we estimate $\prod_{i=1}^{s}\left(1-q^{i}\right)$ by $\phi(q)$. Accordingly, we can conclude that a.a.s., $\operatorname{dim}(P) \leq d$ if

$$
E[X]=n m q(\phi(q))^{r} \rightarrow 0 .
$$

We note that $\phi(q)<1, r=d / n=n / m-1$ and $p=1-q \leq 1 / 2$. It follows that inequality (17) is equivalent to:

$$
n m q=o\left(e^{n \log (1 / \phi(q)) / m}\right) .
$$

With $z$ set at $n^{2} q \log (1 / \phi(q)$, we note that $z$ and $\log z$ tend to infinity with $n$. Now set $m=n \log (1 / \phi(q)) / \log z$. Since $e^{\log z}=z=n^{2} q \log (1 / \phi(q))$, inequality (18) is equivalent to:

$$
n^{2} q \log (1 / \phi(q))=o\left(n^{2} q \log (1 / \phi(q)) \log z\right) .
$$

Clearly, this last inequality is satisfied.

We are reasonably confident that New Upper Bound (3) is asymptotically correct.

Lemma 5.4 (New Upper Bound (2)). If $n^{-1 / 2} \log n<q \leq n^{-1 / 3}$, then a.a.s., $\operatorname{dim}(P)<n-q n /(2 \log (q n))$.

Proof. We note that for all pairs $(m, r)$, there is a $(m, r, 1)-$ GLR. Of course, this simply means that we put every element of $M$ in second position $d / m=r$ times. Setting $s=1$ in equation (16), we can conclude that a.a.s., $\operatorname{dim}(P) \leq d$ if

$$
n m q(1-q)^{d / m} \rightarrow 0 .
$$

Again, we note that $(1-q)^{d / m}=(1-q)^{n / m} /(1-q) \geq(1-q)^{n-m} / 2$. Now set $m=q n /(2 \log (q n))$. Noting that $2 \log (q n) \rightarrow \infty$, we have

$$
(1-q)^{n / m}=e^{-q n / m}=e^{2 \log (q n)}=n^{2} q^{2} .
$$

Therefore, inequality (20) is equivalent to:

$$
n^{2} q^{2}=o\left(\log (q n) n^{2} q^{2}\right) .
$$

This last equation holds since $q n \geq \log ^{2} n$ so that $\log (q n) \rightarrow \infty$.

\section{Applications to the Extremal Problems}

We now return to Question 1 and the problem of finding the correct exponent on the function $\mathrm{sa}(c)$. Previously, we reported that we had been able to use the asymmetric form of the Lovász local lemma to raise the lower bound on the exponent of $c$ in $\operatorname{sa}(c)$ from $4 / 3$ to $3 / 2$. This unpublished result was presented at several conferences and seminars and was proved by using the local lemma to find a rare poset with independence number less than 2 and only moderately large standard example number. Lemma 2.3 was then used to determine the dimension of such a poset.

However, our new bounds allow us to obtain a simple proof of this same improvement. Consider the value $q=n^{-1 / 3}$. If a bipartite poset $P \in \mathbb{B}(n)$ contains 
the standard example $S_{d}$, then its clique size is at least $d / 2$. Since the clique size of $P \in \Omega(n, p)$ is a.a.s., less than $3 q^{-1} \log (q n)=2 n^{1 / 3} \log n$, it follows that a.a.s., $\operatorname{se}(P)<4 n^{1 / 3} \log n$. On the other hand, with $q=n^{-1 / 3}$, we know that a.a.s.,

$$
\operatorname{dim}(P) \geq n-32(n \log n / q)^{1 / 2}=n-32 n^{2 / 3} \log ^{1 / 2} n .
$$

Setting $c=32 n^{2 / 3} \log ^{1 / 2} n$, the upper bound on $\operatorname{se}(P)$ forces $f(c)=\Omega\left(c^{3 / 2} / \log ^{3 / 4} c\right)$.

6.1. Progress on Question 2 for Posets. We begin with the following elementary result, for which we only outline the proof. Ironically, when applied it will be for $p<1 / 2$.

Lemma 6.1. Suppose $n^{-1} \log ^{2} n \leq q \leq 1 / 2$. Then a.a.s., $A$ and $A^{\prime}$ can be matched.

Proof. Clearly, it is enough to prove the lemma when $q=n^{-1} \log ^{2} n$. The basic idea is to show that a.a.s., Hall's matching condition is satisfied. For a subset $W \subset A$, let $N(W)$ consist of all elements of $A^{\prime}$ that are incomparable with at least one element of $W$. We want to show that a.a.s., $|N(W)| \geq|W|$ for every subset $W \subseteq A$. First, we take care of the case when $W$ is very small or very large.

Set $r=\log n / 5$. Consider the events $E_{1}$ that holds if there is some $a \in A$ incomparable with fewer than $r$ elements of $A^{\prime}$. Dually, event $E_{2}$ holds if there is some element $a^{\prime} \in A^{\prime}$ incomparable with fewer than $r$ elements of $A$. Simple counting shows that $\mathbb{P}\left(E_{i}\right)=o(1)$ for $i=1,2$.

Now consider the event $F$ that holds if there is some set $W$ with $r \leq|W| \leq n-r$ such that $\mid(N(W)|<| W \mid$. Then we show that $\mathbb{P}(F)=o(1)$. Readers will note that this part of the proof is very similar to the proof (which is provided) of Lemma 3.1

Once we have shown that $\mathbb{P}\left(E_{1}\right)=\mathbb{P}\left(E_{2}\right)=o(1)$ and $\mathbb{P}(F)=o(1)$, the proof is complete.

Recall that $f(d, n)$ is the maximum value of $\operatorname{dim}(P)$ among all posets on $n$ points with standard example size less than $d$.

Theorem 6.2. For all $d \geq 3$,

$$
f(d, n) \geq \frac{n^{1-\frac{2 d-1}{d(d-1)}}}{8 \log n} .
$$

Proof. Fix a value of $d \geq 3$. We work in the space $\Omega(n, p)$ with

$$
p=n^{-\frac{2 d-1}{d(d-1)}} \text {. }
$$

We note that $p<1 / 2$. Now set

$$
\epsilon=\frac{2 d(d-1)}{2 d-1}-2 \text { and } t=2 \log n / p
$$

We note that $\epsilon>0$.

Using Lemma 6.1, we know that a.a.s., the sets $A$ and $A^{\prime}$ can be matched. Furthermore, the following claim is just Lemma 3.3 stated in complementary form.

Claim 1. For every $\epsilon$ with $0<\epsilon<1$, a.a.s., $\operatorname{bin}(P)<(2+\epsilon) \log (p n) / p$.

Therefore, a.a.s.,

$$
\operatorname{bin}(P)<(2+\epsilon) \log (p n) / p=(2+\epsilon) \frac{(2 d-1) \log n}{(d-1) p}=2 \log n / p=t .
$$

Next, we need the following technical claim. 
Claim 2. If $P$ is a poset in $\mathbb{B}(n)$ and $\operatorname{bin}(P)<t$, then $P$ does not contain a bipartite subposet $Q=V \cup V^{\prime}$ with $|V|=\left|V^{\prime}\right|=2 t$ satisfying the following condition: The elements of $V$ and $V^{\prime}$ can be labeled as $V=\left\{v_{1}, \ldots, v_{2 t}\right\}$ and $V^{\prime}=\left\{v_{1}^{\prime}, \ldots, v_{2 t}^{\prime}\right\}$ such that there is a linear extension $L$ of $Q$ with $v_{i}>v_{i}^{\prime}$ in $L$ for each $i \in[2 t]$.

Proof. We argue by contradiction. After a relabeling, we may assume that $v_{1}>$ $\cdots>v_{2 t}$ in $L$. However, this implies that $v_{i}>v_{j}^{\prime}$ in $L$ whenever $1 \leq i \leq t$ and $t+1 \leq j \leq 2 t$, which implies that $\operatorname{bin}(P) \geq t$.

Next, we let $X$ be the random variable that counts the number of copies of the standard example $S_{d}$ in $P$. Then the expected value of $X$ is given by

$$
\mathbb{E}[X]=\left(\begin{array}{l}
n \\
d
\end{array}\right)^{2} d !(1-p)^{d} p^{d(d-1)}<\frac{n^{2 d}}{6} p^{d^{2}-d}=n / 6
$$

Let $E$ be the event that occurs when $X>n / 4$. Then $\mathbb{P}(E)<2 / 3$. It follows that there is a poset $P \in \Omega(n, p)$ such that (1) $A$ and $A^{\prime}$ can be matched; (2) $\operatorname{bin}(P)<t$; and (3) the number of copies of the standard example $S_{d}$ in $P$ is at most $n / 4$. Let $A=\left\{a_{1}, a_{2}, \ldots, a_{n}\right\}$ and $A^{\prime}=\left\{a_{1}^{\prime}, a_{2}^{\prime}, \ldots, a_{n}^{\prime}\right\}$ be labelings that evidence a matching between $A$ and $A^{\prime}$. Without loss of generality, we may assume that any copy of $S_{d}$ contained in $P$ (there are at most $n / 4$ of them) contains some point in $\left\{a_{i}: n / 2<i \leq n\right\} \cup\left\{a_{i}^{\prime}: n / 2<i \leq n\right\}$. Hence there are no copies of $S_{d}$ in the bipartite subposet $Q=B \cup B^{\prime}$ where $B=\left\{a_{i}: 1 \leq i \leq n / 2\right\}$ and $B^{\prime}=\left\{a_{i}^{\prime}: 1 \leq i \leq n / 2\right\}$. Note that we have $|Q|=n$.

Now let $\mathcal{F}$ be any family of linear extensions of $Q$ which is a realizer of $P$. Then $\mathcal{F}$ must reverse the pairs in $\left\{\left(a_{i}, a_{i}^{\prime}\right): 1 \leq i \leq n / 2\right\}$. However, in view of the claim, no linear extension can reverse $2 t$ of these pairs. we conclude that

$$
\operatorname{dim}(P) \geq \operatorname{dim}(Q) \geq \frac{n}{4 t}=\frac{p n}{8 \log n}=\frac{n^{1-\frac{2 d-1}{d(d-1)}}}{8 \log n} .
$$

\section{Some Comments on Open Problems}

We view the problem of determining the expected value of $\operatorname{dim}(P)$ for posets in $\Omega(n, p)$ when $n^{-4 / 5}<q \leq n^{-1 / 3}$ to be a real challenge. We suspect that our New Upper Bounds are near the truth, but we cannot rule out the possibility that for almost all $P$, if the short dimension of $P$ is $d$ as evidenced by a short realizer $\Sigma$ of size $d$, then $\mathbb{P}(\Sigma(R))$ is very small.

Second, although we believe we know the expected value of $n-\operatorname{dim}(P)$ to within a $1+o(1)$ multiplicative factor when $1 / 2 \leq p<1-n^{-1 / 3}$ as specified by New Upper Bound (1), the challenge is that there are other constructions besides generalized latin rectangles that achieve the same bound.

For the first extremal problem, stability analysis, we continue to think it likely that the correct exponent for $c$ in the function $\operatorname{sa}(c)$ is 2 . As we have noted, this would be verified if the upper bound on $\operatorname{dim}(P)$ when $q=n^{-1 / 2}$ is correct to within a poly-log multiplicative factor on $n-\operatorname{dim}(P)$. Alternatively, one could revisit the proof given in 3] and try to lower the exponent in the inequality $\operatorname{sa}(c)=O\left(c^{2}\right)$. Success in this effort would of course imply that our upper bounds on $\operatorname{dim}(P)$ are not as good as we think. 
For the second extremal problem, it would be very interesting to show that for each $d \geq 3$, there is a constant $c_{d}$, with $0<c_{d}<1$, such that $f(n, d)<n^{c_{d}}$, although it is not clear that such a constant exists, even when $d=3$.

\section{Acknowledgment}

The authors would like to thank Noga Alon, Alan Frieze, and Tomasz Łuczak for very helpful communications concerning second moment methods, the concept of defect, and Talagrand's inequality. As noted previously, the heart of the proof of Lemma 3.5 was provided by Luczak in a personal communication.

\section{REFERENCES}

[1] N. Alon and J. Spencer, The Probabilistic Method, 4th Edition, Wiley Series in Discrete Mathematics and Optimization, 2016.

[2] C. Biró, P. Hamburger and A. Pór, Standard examples as subposets of posets, Order 32 (2015) 293-299. doi:10.1007/s11083-014-9331-y

[3] C. Biró, P. Hamburger, A. Pór and W. T. Trotter, Forcing posets with large dimension to contain large standard examples, Graphs and Combinatorics 32 (2016) 861-880. doi:10.1007/s00373-015-1624-4

[4] B. Dushnik and E. W. Miller, Partially ordered sets, Amer. J. Math. 63 (1941), 600-610. doi: $10.2307 / 2371374$

[5] P. Erdös, H. Kierstead and W. T. Trotter, The dimension of random ordered sets, Random Structures and Algorithms 2 (1991), 253-275. doi:10.1002/rsa.3240020302

[6] P. Erdős and L. Lovász, Problems and results on 3-chromatic hypergraphs and some related problems, in Finite and Infinte Sets, A. Hajnal, R. Rado and V. T. Sós, eds., North Holland, 1975, 609-625. web.cs.elte.hu/ lovasz/scans/LocalLem.pdf

[7] A. M. Frieze, On the independence number of random graphs, Discrete Math. 81 (1990), 171-175. doi:10.1016/0012-365X(90)90149-C.

[8] Z. Füredi and J. Kahn, On the dimension of ordered sets of bounded degree, Order 3 (1986), 15-20. doi:10.1007/BF00403406

[9] T. Hiraguchi, On the dimension of orders, Science Reports of the Kanazawa University 4 (1955), 1-20. scirep.w3.kanazawa-u.ac.jp/articles/04-01-001.pdf

[10] S. Janson, T. Łuczak and A. Rucinski, Random Graphs, Wiley, New York, N.Y., 2000.

[11] R. J. Kimble, Extremal Problems in Dimension Theory for Partially Ordered Sets, Ph.D. thesis, Massachusetts Institute of Technology, 1973. dspace.mit.edu/bitstream/handle/1721.1/82903/30083917-MIT.pdf

[12] A. Scott and D. Wood, Better bounds for poset dimension and boxicity, Transactions Amer. Math. Soc., to appear. Available on the arXiv at 1804.03271

[13] W. T. Trotter, Problems and conjectures in the combinatorial theory of ordered sets, Annals Discrete Math. 41 (1989), 401-416. doi:10.1016/S0167-5060(08)70478-3

[14] W. T. Trotter, Dimension for posets and chromatic number for graphs, in 50 Years of Combinatorics, Graph Theory and Computing, Chung et al., eds., to appear. 
Department of Mathematics, University of Louisville, Louisville, Kentucky 40292

E-mail address: csaba.biro@louisville.edu

Purdue University, IN,

E-mail address: hamburge@pfw.edu

School of Mathematical and Statistical Sciences, Arizona State University, Tempe, ARIZONA 85287

E-mail address: kierstead@asu.edu

Department of Mathematics, Western Kentucky University, Bowling Green, KenTUCKY 42101

E-mail address: attila.por@wku.edu

School of Mathematics, Georgia Institute of Technology, Atlanta, Georgia 30332

E-mail address: trotter@math.gatech.edu

School of Mathematics, Georgia Institute of Technology, Atlanta, Georgia 30332

E-mail address: rwang49@math.gatech.edu 